\title{
The Coherent Beam-Beam Interaction
}

\author{
S. Krishnagopal* \\ Lawrence Berkeley Laboratory, University of California, Berkeley, California 94720 \\ R. Siemann"* \\ Stanford Linear Accelerator Center, Stanford, CA 94305
}

May 1991

"This work was supported by the Director, Office of Energy Research, Office of High Energy and Nuclear Physics, Division of High Energy Physics, of the US Department of Energy under contract DE-AC03-76SF00098.

"* Supported by the Office of Energy Research, U.S. Departmert of Energy, under Contract No. DE-AC03-76SF00515 


\title{
The Coherent Beam-Beam Interaction
}

\author{
S. Krishnagopal $\left({ }^{*}\right)$ \\ Lawrence Berkeley Laboratory, \\ Berkeley, CA 94720 \\ R.Siemann $\left({ }^{* *}\right)$
Stanford Linear Accelerator Center,
Stanford, CA 94309
}

\section{Abstract}

We present the results of a new beam-beam simulation program that allows for a self-consistent calculation of the electromagnetic fields of the beams by treating general (non-Gaussian) beam-distributions. We find that a new class of colserent instabilities, appearing at certain operating points, dominate the dynamics.

\section{INTRODUCTION}

One of the factors limiting the performance of $e^{+} e^{-}$storage ring colliders is the beam-beam interaction. There has been much speculation on the role of colherent (or collective) beam-beain effects as a mechanism for limiting the tune-slift, but no consensus has been reached on this issue[1]. Centroid (or dipole) motion, where the centroids of the two beams oscillate relative to each other, is routinely observed in operating storage rings, but there is no evidence that it affects luninosity. Centroid motion is easily detected and could be removed with feedback.

The potential for performance linitations comes from effects that distort the beam shape. Such effects have been analyzed with two different types of models. In the first, of Furman et al.[2] and of Hirata[3], nonlinear maps for the colliding beam system are developed in the moments. In earlier work Hirata uses a nonlinear beam-beam kick calculated from a Gaussian beam, which is not consistent with the assumption that the beants remain Gaussian. He finds flip-flop solutions, where both beams maintain unequal sizes. Later attempts at including higher moments were in substantial agreement with the Gaussian calculation. Furman et al. maintain self-consistency at the cost of a simplified, linear model of the beam-beam force. They find that equal-size higher-period solutions coexist, and are responsible for performance linitations.

In the second type of model, of Chao and Ruth[4] and of Dikansky and Pestrikov[5], the pliase space distributions of the two bearis influence each other and modes develop in phase space. The stability of these modes is analyzed with the linearized Vlasov equation, assuming small perturbations from equilibrium. While these calculations indicate the potential importance of coherent beam-beam effects, there are open questions about the approximations used in the calculations, Landau and radiation damping, and the relative importance compared to ol :er effects.

Strong-strong compucer simulations are an important tool in the study of the coherent beam-beam interaction. In the usual 'Gaussian simulations' the positions and rms sizes of the beams are first calculated from the coordinates of the test-particles just before the collis on. They are then used in an expression for the beam-b am force that assumes the particle distribution to be Gaus an[6]. Unfortunately, this procedure does not allow for a . If-consistent calculation of the fields; it restricts the fields directly and the distributions implicitly.

The simulation program discussed in this paper calculates the fields from the coordinates of the test-particles, and does not impose any restrictions on the beamdistributions or the beam-profiles. We find new coherent instabilities that, at certain operating points, dominate the dynamics.

\section{FIELD CALCULATION}

We worked with beams that were nominally round, i.e. the $\beta^{*}$ 's and nominal emittances were equal in the two transverse dimensions. The beams were not restricted to remain round[7], but the field calculation is most efficient when the transverse sizes are comparable.

The electromagnetic fields of a bunch are calculated by Lorentz transforming to its rest frame and then solving Poisson's equation. Test particles are cast onto a circular mesh with radial step size $\Delta r$ and azimuthal bin size $\Delta \phi$. The array that results, $N(r, \phi)$, must be smoothed, otherwise the simulation results are affected by statistical fluctuations due to the finite number of test particles. The average charge is treated first. It is smoothed by least-squares fitting using Forsythe's method[8], and the radial electric field it produces is calculated using Gauss' Law. The azimuthal variation of $N(r, \phi)$ is treated by Fourier analysing $N(r, \phi)$ and checking each Fourier coeffi. cient for consistency with zero. Statistically significant coefficients are fitted and smoothed with Forsythe's method, and the Green's function for Poisson's equation in polar coordinates[9] is used to calculate the potential and the electric field. 


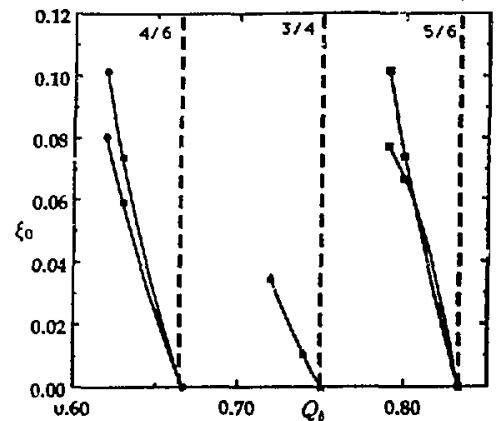

Figure 1: Onset and offset $\xi_{0}$ for the various coherent resonances, as a function of $Q_{b}$. At the nominal tune of the resonance it is assumed that the resonauce 'tube' vanishes. The lines are drawn by interpolating from the calculated data points down to the zero- $\xi_{0}$ point.

This algorithm was checked for sensitivity to the details of the mesh - the sizes of $\Delta r$ and $\Delta \phi-$ and was found to be insensitive to them. On the other hand, the number of test particles and the smoothing provided by the least squares fitting were important. Witlout smoothing, statistical fluctuations dominated for any reasonable number of test particles. With smoothing, results were found to be insensitive to the number of test particles when that number exceeded 5,000; in this work we used 10,000 test particles.

\section{SIMULATION RESULTS}

Our results show that the coherent behaviour is sensitive to the radiation damping. In this work we concentrated on low-order resonances, up to sixth order, and on damping decrements of $\delta=1 \times 10^{-3}$ and $\delta=1 \times 10^{-4}$, corresponding to betatron damping times of 2,000 and 20,000 turns, respectively. Synchrotron motion was not included, and the beams were forced to collide head-on by a 'feedback' system that set the centroids of the beams to zero after each turn. The borizontal and vertical tunes were kept equal ( $=Q_{b}$, say), and $Q_{b}$ was restricted to the region $0.5<Q_{b}<1.0$. Results are presented only for the $x$ dimension; the $y$-dimension behaved analogously.

\section{A Tune-Shift Scans}

Figure 1 presents the results of scans over the nominal tune-slifft $\xi_{0}$, performed at different tunes, in order to determine the tune-sbift region over which coherent motiun broke out. The data-points in the figure correspond the lowest and highest $\xi_{0}$ 's at which coherent motion was seen, at that particular tune.

For the fourlh-order $\left(\frac{3}{4}\right)$ resonance there seems to be no upper limit to the coherent motion, at least to the max-

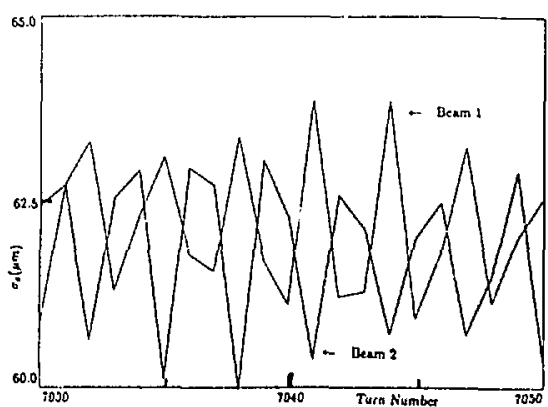

Figure 2: Horizontal beam-size variation over 20 turns. $Q_{b}=0.79, \xi_{0}=0.08$.

imum $\xi_{0}$ of 0.14 investigated. A study of the beam-size variation indicates that the behaviour is period-2 and anticorrelated between the two beams; i.e. when one beam is tightly focussed, the other is blown-up. This is consistent with the results of Furman et al.[2] which show a strong fourth-order resonance. This is not a new feature of these simulations; the same behaviour is seen even in Gaussian simulations.

Two sixtli-order resonances, the $\frac{4}{6}$ and +?e $\frac{5}{6}$, wer 2 identified and traced out in tune. In contrast to the $\frac{3}{4}$ resonance, they were found to have a finite width in $\xi_{0}$. This is consistent with the predictions of the second class of models discussed above $[4,5]$. The oscillations in the beam-sizes were found to be period-3 and anti-correlated (Fig. 2).

Just below $Q_{b}=0.667$ both third $\left(\frac{2}{3}\right)$ and $\operatorname{sixth}\left(\frac{4}{6}\right)$ order resonances could play a role in the dynamics. On the other hand below $Q_{b}=0.833$ only the sixth order $\left(\frac{5}{6}\right)$ resonance is possible. However, the similarity of the coherent dynamics in these two regions ( $F ;$ 1) suggests that the thirdorder resonance does not contribute below $Q_{0}=0.667$. More generally one may surmise that odd order coherent resonances do not occur at all. This agrees with the predictions of the second class of models $[4,5]$, and is significant from the practical point of viev.

\section{$B$ Gaussian vs General Simulations}

To slow that the sixth-order resonances are a new feature of these simulations, we performed a set of comparison runs with a Gaussian sinulation. For the $\frac{5}{6}$ resonance, at a tune of $Q_{b}=0.80$, the region over which coherent motion was seen was scanned using a Gaussian simulation.

The results are presented in Fig. 3 in terms of the beamsize and its variation as a function of $\xi_{0}$ for both, the general and the Gaussian simulations. For the former, with 10,000 test particles, the statistical fluctuation in the beam-size is less than a micron. For the latter, with 1,000 particles, it is just over a micron. Larger size-variations are indicative of colerent motion. 


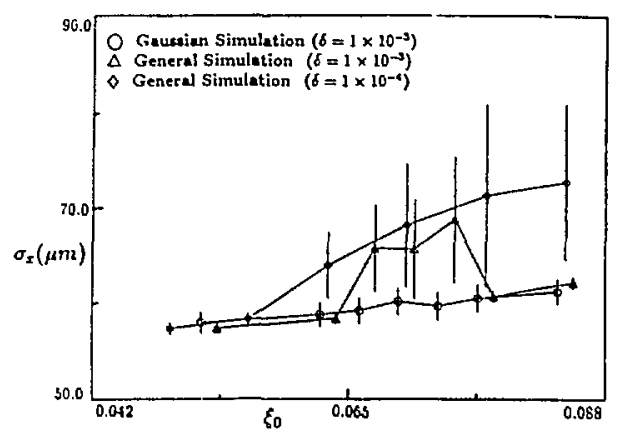

Figure 3: Horizontal beam-size as a function of $\xi_{0}$ at $Q_{b}=$ 0.80 , for three different cases.

From the figure oue sees that for the sixth-order resonance coherent oscillations are present only in the more general simulation; they are absent in the Gaussian simulation.

\section{DISCUSSION}

The last observation above suggests that it is critical tr. use general field calculations in the study of nonlinear coherent resonances. The reason is that this allows for a selfconsistent calculation of the electromagnetic fields from the positions of the test particles. It results in higher-order fixed-point coherent motion.

It should be noted that centroid motion has been removed in these simulations, allowing us to concentrate on quadrupolar coherent oscillations. This is also reasonable from the experimental point of view, because such motion could be removed using feedback systems. If it is allowed to remain, we find that the beam-size variations persist, although they are reduced in magnitude. There is now a large degree of centroid motion between the beams. Thus both centroid and shape uscillations take place, and the situation is complex.

As mentioned earlier, the coherent behaviour is sensitive to the damping decrement $\delta$. With lesser damping the width of the resonance becomes considerably larger, as can be seen from Fig. 3. Further, this could potentially result in ligher-order resonances entering the picture; some prelininary results with $\delta=1 \times 10^{-4}$ indicate that this is indeed the case. Thus, in machines with low radiation damping these coberent colierent resonances could play an important role.

It should be emphasized that the signature of these coherent resonances is a swift, turn-to-turn, variation in the beam-sizes. Since existing detectors are not sensitive to such rapid variations, these resonar.ces could not, hitherto, have been detected.

\section{FUTURE WORK AND CONCLUSIONS}

Mucl work remains to be done, and is in progress. Resonance structure with lower damping is being investigated. The impact of removing feedback has to be studied. Sensitivity to initial conditions, consequences of asymmetric parameters between the two beams, and methods of handling flat beams; all these avenues can be explored.

We conclude by observing that a self-consistent calculation of the electromagnetic fields is essential in a study of the colierent beam-beam interaction. It leads to a new class of higher-period coherent instabilities that dominate the dynamics at certain operating points.

This research was conducted using the Cornell National Supercomputing Facility, a resource of the Center for Theory and Simulation in Science and Engineering at Cornell University, which is funded in part by the National Science Foundation, New York State and the IBM Corporation. Early work on this topic was performed at the Laboratory of Nuclear Studies, Cornell University which is supported in part by the National Science Foundation.

$\left(^{*}\right)$ Work supported by the Department of Energy, contract DE-AC03-76SF00098.

$\left(^{*}\right)$ Work supported by the Department of Energy, contract DE-AC03-76SF00515.

\section{REFERENCES}

[1] Working group sunımaries in the Proceedings of the Third Advanced ICFA Ream Dynamics Workshop on Beam-Beam Effects in Circular Colliders, (Novosibirsk: Inst. of Nucl. Ply's., 1989), edited by I.Koop and G.Tumaikin.

[2] M.A.Furman, K.Y.Ng and A.W.Chao, SSC-174, (SSCL, 1988).

[3] K.Hirata, Phys.Rev.Lett. 58, 25 (1987); 58, 1798 (E) (1987); Pliys. Rev. D37, 1307 (1988); AIP Conf. Proc. 214, 175 (1990).

[4] A.W.Chao and R.D.Ruth, Part. Accel. 16, 201 (1985).

[5] N.S.Dikansky and D.V.Pestrikov, Part.. Accel. 12, 27 (1982).

[6] M.Bassetti and G.A.Ershine, CERN-ISR-TH/80-06, (1980).

[7] Earlier work with this restriction is contained in: S.Krislunagopal, Ph.D. Dissertation, Cornell University (1991). There too, the same resonances occur.

[8] L.G. Kelly, Ilandbook of Nunerical Methods and Applications (Reading, Mass: Addison-Wesley, 1967), p. 68.

[9] P.M. Morse and II. Feshbach, Methods of Theoretical Physics (New York: McGraw-Hill, 1953), p. 1188. 